\title{
LOCAL SHEEP SKELETON PREPARATION: DEVELOPMENT OF A NEW PRESERVATION TECHNIQUE
}

\author{
RONAK SABER HABIB \\ Dept. of Theriogenology, Physiology \& Anatomy, College of Veterinary Medicine, University of \\ Duhok, Kurdistan Region-Iraq
}

(Received: July 21, 2019; Accepted for Publication: September 23, 2019)

\begin{abstract}
Cracking, crumbling, non-pleasant smell and changing bone color during and after bone preservation are the most common issues in the former skeleton models' preparation.

This qualitative-experimental project presents a new technique by mixing between a number of the old techniques of preservation of the skeleton models via the below four main steps: First, boiling the bones with tap water and degreasing with a detergent. Then, drying naturally under sunlight. Afterward, boiling with $3 \%$ hydrogen peroxide. Finally, re-attaching and installation of the final skeleton model on a wooden plate.

The vertebrae was articulated with each other in their main anatomical positions. The ribs were fixed with the thoracic vertebrae. The mandible was attached to the skull, and the skull was installed into the vertebral column. The fore- and hind-limbs were assembled; the scapula was attached with the ribs and the femur with the pelvic bone at the acetabulum. Finally, the carpal, tarsus, patella, and sesamoid bones were assembled and fixed in their main anatomical positions. The final installed skeleton formed with desirable white color, with no foul smell, no crack and crumble and highly tolerable to cold and hot temperature.

The present study Proposed that prepared sheep skeleton model can be used to promote the veterinary students' comprehension for the anatomy courses since the method has been used in this study resulted in a high-quality skeleton model in its color, texture and tolerability with no irritant foul smell.
\end{abstract}

KEYWORDS: Sheep; Skeleton Model; Bones; Detergent; hydrogen peroxide. https://doi.org/10.26682/ajuod.2019.22.2.8

\section{INTRODUCTION}

$S$ keleton models are very important and essential in anatomy halls and museums to promote anatomy comprehension, which is considered the most important course in the preclinical stages (Bloom, 1956; Hafsa and Stanek, 2007). Skeleton gives students a handson method for learning bone names and locations, as well as a visual tool to relate bone and joint structure to muscle structure (Greene et al., 1993). There are different techniques or methods used in bone preparation, which including leaving bones above or burying in the ground, insect consumption, cold- or warmwater maceration, as well as boiling in hot water. Other methods of bone preparation are the introduction of enzymes or chemicals to fasten the removal of the attached soft tissues from the bones (Allouch and Al-sheikh, 2008).

The preservation of bones is usually processed through several chemical and mobility steps included boiling, soaking, and completion steps (Allouch and Al-sheikh, 2008; Hussain et al., 2007). The easiest way to remove bone grease was to soak the bones in ammonia (Gram, 2006). Preparation of bones involves soft tissue removal or bone cleaning, bone bleaching, bone articulation, and labeling. Depending on the size of the animal, each of the preceding processes may vary in the amount of time required (Allouch and Al-sheikh, 2008). The available skeleton models, most of the times, have a nonpleasant smell because of degreasing technique in their preservation methods or sometimes they crack, crumble and changing in the bone color due to different reasons, such as high concentration of $\mathrm{H} 2 \mathrm{O} 2$, or boiling time.

The purpose of this study was to prepare a real skeleton model of a small ruminant to teach the osteology and comparative anatomy of bones. We expect that our skeleton preservation method could overcome the previous 
disadvantages of the former techniques which is mentioned above.

\section{SUBJECT AND METHODS \\ Study Design:}

This qualitative-experimental study was to prepare a real skeleton model of a domestic sheep, which is consisting of approximately 215 individual bones rigidly connected. Among all the species and different type of animals, local sheep were selected based on the below criteria:

First, laboratory requirement: Anatomy lab at College of veterinary Medicine- University of Duhok was at high demand for this kind of model to take a benefit for teaching and educational purposes. Then, a typical example: sheep represent a typical example of the eventoed ungulates. Finally, easy to handle: sheep are animals that are very easy to deal with before and after slaughtering with affordable price.

\section{Materials and Method:}

In our study bones were prepared to be a real model skeleton using boiling water method, degreasing by using a detergent, hydrogen peroxide $\left(\mathrm{H}_{2} \mathrm{O}_{2}\right)$ to bleach the bones, a special super-adhesive glue to fix the bones in their anatomical location, and eventually install the model on a specified wooden plate; this was done based on the following main steps:

\section{Sampling:}

1. Choosing the animal: A local female sheep (ewe) has been selected.

2. Slaughtering: the slaughtering process was done by the appropriate Islamic method.

\section{Bone Preparation:}

1. Skinning and cleaning: removing the skin and internal organs, then cleaning thoroughly by tap water.

2. Removing flesh: removing flesh mass on the bones to separate the animal body into different parts.

3. Separating bones/ body parts: The separated bones were: skull, fore-limb, hind-limb, vertebrae, ribs \& sternum, and pelvic bone.

\section{Bone Preservation:}

1. Boiling the separated bones: boiling the bones in a mixed solution of tap water and a washing powder detergent for maximum 6 hours to loosen the flesh around the bones and also, the bones remain in their main shapes, density and rigidity.

2. Complete individual bone cleaning: after boiling, bones have been relocated and separated from the joints carefully, and cleaned thoroughly from all the remaining flesh.

3. Drying: the bones have been exposed to sunlight for one day until get dry completely.

4. Bone Bleaching: it was done by boiling the dried bones in a diluted solution of $3 \%$ hydrogen peroxide (H2O2) for 15 minutes and left in the solution for one week after that is drying them under the sunlight for one day.

\section{Assembling:}

At this very final step, we were collecting the bones and re-attaching them to their main anatomical position by a strong super-adhesive glue, and fixed and installed the skeleton model on a specified wooden plate.

The skeleton was mounted on a wooden base plate, length: $20 \mathrm{~cm}$, width: $40 \mathrm{~cm}$, height at withers: $70 \mathrm{~cm}$, weight: $60 \mathrm{~kg}$; also supported by two stainless steel rods to carry the animal's body, the first at the temporal bone of skull, and the second at the level of last thoracic vertebrae and first lumbar vertebrae which gave the desired strength and support.

\section{RESULTS}

The vertebral column was shaped by arranging the cervical, thoracic, lumbar, sacral and caudal vertebrae fixing them with superadhesive glue. Moreover, the articular surfaces were pasted with each other by adhesive material from the first cervical to the first sacral vertebrae to give the vertebral column more support and rigidity (Fig. A). On the other hand, the ribs were fixed with the thoracic vertebrae by adhesive glue. The ending of the sternal ribs were attached by tapes of the plastic hook (Fig. B).

The mandible was attached to the skull via the condyloid process to the temporal bone by adhesive glue. Moreover, the mandibular teeth were adhered with the upper jaw to increase the connectivity, durability, and strength. The skull was installed to the vertebral column by entering it within the magnum foramen. Moreover, plastic hook was used to connect between magnum foramen of the occipital bone and the first cervical vertebra (Fig. C). The fore- and hind-limbs were assembled by placing the bones in the correct conformation. The holes of the proximal and distal extremities of the long bones were placed sequentially in diameter to take their real shapes (Fig. D). 
The super-adhesive glue attached the scapula with ribs. Moreover, the femur was attached with the pelvic bone at the acetabulum by using an adhesive material to give it support and strength (Fig. E). Finally, the carpal, tarsus, patella, and sesamoid bones were assembled and fixed with each other by super-adhesive glue (Fig. F).

Our skeleton model was installed successfully in anatomy lab, and it was exposed to the various low and high temperature, minimum $4{ }^{\circ} \mathrm{C}$ to maximum $40{ }^{\circ} \mathrm{C}$, from March 2019 to July 2019 , to check the tolerability of the preserved bone and to record any change in the color and texture.

We observed that the local sheep skeleton model prepared by this new technique did not change its original color and has been preserved with perfect bleaching, and the method we used mask the later irritant foul smell and cracked bone that was occurred in previous method and models.

\section{DISCUSSION}

All experiments in this study were completed without any complications regarding the animals' bone: their shapes, density, rigidity, and color. The present study revealed that the skeleton model's preservation technique of small animals improves the teaching of anatomy courses. The main four steps of preservation technique used in this study, including boiling with water, degreasing with a detergent, naturally drying under sunlight, and bleaching with hydrogen peroxide, based on the experimental timing of each step and concentrations of solutions are the successful methods with desirable results. Greene et al., (1993) and Hussain et al., (2007) reported that the large animals (camel, horse, and ostrich) must be boiled for 8 hours, 2 hours for the poultry and 6 hours for the sheep, reflecting our methods and results. Extra boiling of the flat and thin bones (skull and ribs) led to disintegration and fragility of the bones (Hussain et al., 2007).

In this project, the flesh has been removed from the bone by boiling separated part of the animal body and degreasing by using a washing powder detergent, which we believed that it is the main reason of masking the later foul smell of bones in the previous study and models. Allouch and sheikh, (2008) mentioned that the bones were only prepared previously by boiling bones, in which, the topographic positions of bones and the angles formed between neighboring bones were unknown for the student. Moreover, the boiling with tap water only is caused by rot and texture fatty spam with foul smell later. All of these were uncomfortable for the teachers, students and technicians.

Also, Greene et al., (1993) and Gram, (2006) reported that gasoline $95 \%$ was used for degreasing, because it was very cheap and available. Nevertheless, to avoid irritation from vapors container must be remained strongly closed. They revealed that gasoline has three disadvantages, fast inflammable, ignition, and foul smell. On the other hand, trichloroethylene is excellent degreasing, but it is costly and danger (Tompsett, 1970 and Gram, 2006).

In our study, bones after complete degreasing, cleaning, and drying, has been bleached by boiling with $3 \%$ hydrogen peroxide solution for 15 minutes and then leave in it for a whole week; we believed that the perfect timing for bleaching and drying naturally under sunlight are the most logical reason for excellent whitening of the bones without any cracking and crumbling. In accordance to our method and results, Greene et al., (1993), Gram, (2006), Hussain, et al., (2007) used hydrogen peroxide $3 \%$ solution for the bleaching purposes. Care must be taken for concentration and timing because results showed that increasing timing and concentration of the Hydrogen peroxide led to crumbling the bones (Hussain, et al., 2007).

Strength and limitation: To my knowledge, the preparation of a real model sheep skeleton has not been previously studied nor prepared in our area. We hope that the present study adds useful knowledge to the current gap in veterinary medicine. However, the findings reported in the present study must be interpreted with caution since we could prepare only one model based on our methods and limited materials and budget.

\section{CONCLUSIONS}

The current study proposed and developed a new method to prepare small ruminant skeleton model for teaching purposes, by mixing between a number of the old techniques, such as using boiling water method and washing powder and using hydrogen peroxide solution for bleaching. The current study helped in developing and 
improving the techniques of animal skeleton model preparation and improving the teaching methods of veterinary anatomy in anatomy laboratories.

\section{Acknowledgment:}

My profound thanks and gratitude to my husband, Burhan Abdullah Zaman, for his continuing support and help to complete this study.

\section{Disclosure statement:}

The author reported no potential conflict of interest.

\section{Funding:}

The author was only financial supporter of the study.

\section{Authorship Criteria:}

Author attests she meet the ICMJE criteria for authorship

\section{REFERENCES}

Allouch G \& Al-sheikh kh (2008). Textbook of Comparative anatomy, the bones, ligaments and joints, practical part. Pp 20-22. Veterinary Medicine College, AL Baath University.
Bloom BS (1956). Taxonomy of Educational Objectives, Handbook I: Hildebrand M (1968). Anatomica1 Preparations. pp 16-35. University of Cognitive Domain. Longmans, Green, New York.

Greene EA, Smith KR, Pendergraft JS, Raub RH, and Arns MJ (1993). Technical Note: Equine Skeletal Preservation Techniques to Enhance Teaching Effectiveness. J. Anim. Sci.71:22702274.

Gram CO (2006). Vertebrate Skeletons: Preparation and Storage; pp 7-11 National Park Service.

Hafsa Z and Stanek C (2007). Three Rs in the Research and Education System of Pakistan: Perspectives and Possibilities. Proc. 6th World Congress on Alternatives and Animal Use in the Life Sciences August 21-25, 2007, Tokyo, Japan.

Hussain M, Hussain N, Zainab H, and Qaiser S (2007). Skeletal Preservation Techniques to Enhance Veterinary Anatomy Teaching.IJAVMS Vol. 1, 2007:21-23.

Tompsett, D. H. (1970). Anatomical Techniques. pp 242-247. E. and SS. Livingstone, Edinburgh, U.K.

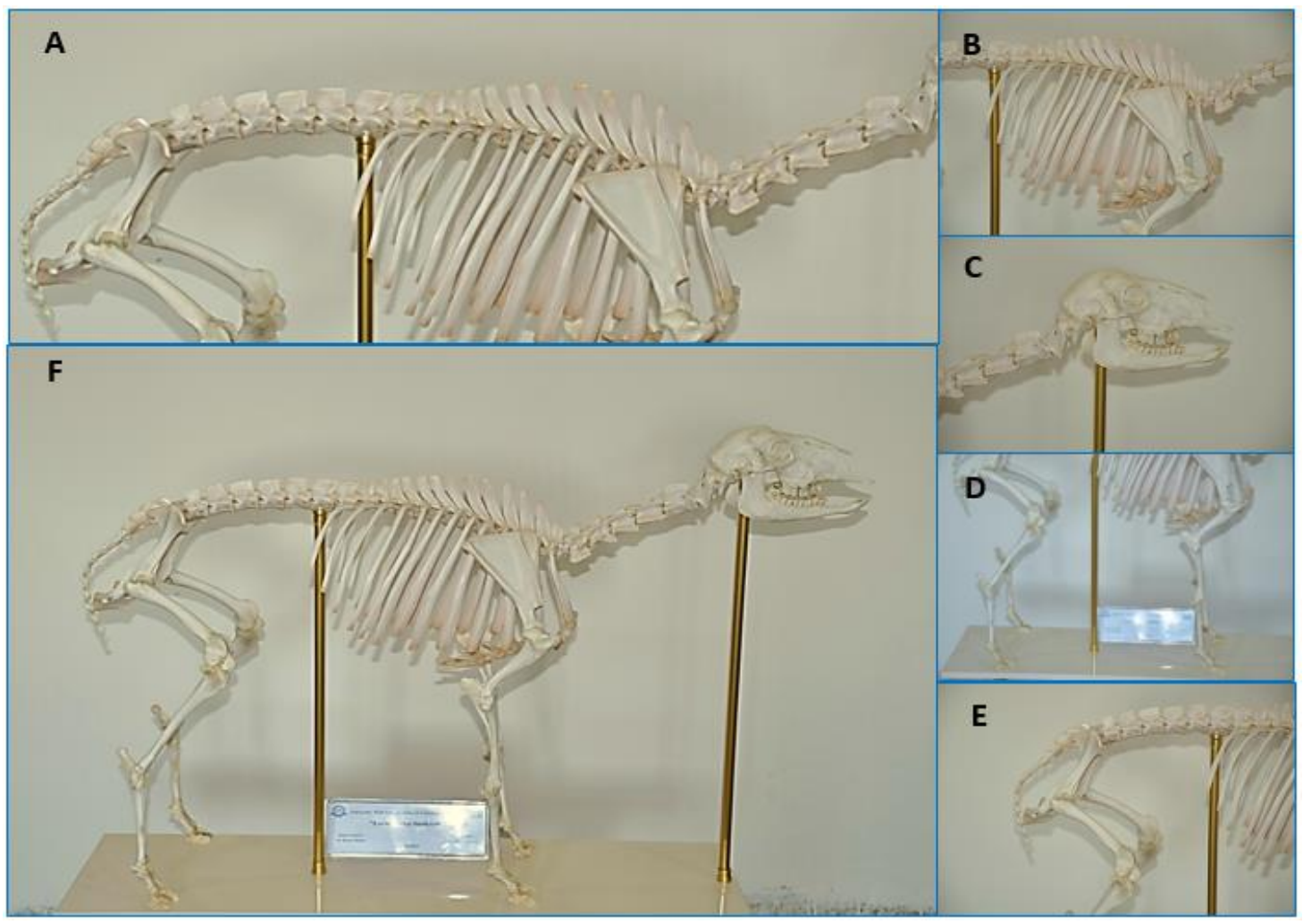

Fig. : A real photo of prepared sheep skeleton model: A. Vertebrae, B. Ribs \& Scapula, C. Skull, D. Fore- \& Hind-limbs, E. Femure, and F. Sheep skeleton model. 